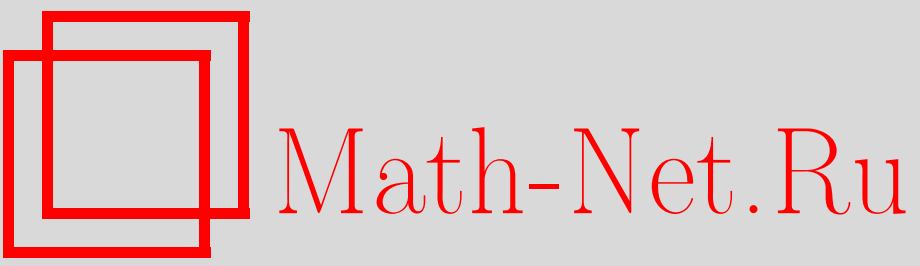

И. С. Тюрин, О скорости сходимости в теореме Ляпунова, Теория вероятн. и ее примен., 2010, том 55, выпуск 2, 250-270

DOI: https://doi.org/10.4213/tvp4200

Использование Общероссийского математического портала Math-Net.Ru подразумевает, что вы прочитали и согласны с пользовательским соглашением

http://www.mathnet.ru/rus/agreement

Параметры загрузки:

IP : 54.197 .217 .227

26 апреля 2023 г., 13:17:32 


\title{
О СКОРОСТИ СХОДИМОСТИ В ТЕОРЕМЕ ЛЯПУНОВА
}

\begin{abstract}
Изучается скорость сходимости в теореме Ляпунова при наличии абсолютных моментов третьего порядка. Применение выпуклого анализа позволило установить неулучшаемую оценку близости в средней метрике вероятностного распределения и его преобразования нулевого смещения. Этот результат применен к оценке точности гауссовской аппроксимации в равномерной метрике, а также метриках $\zeta_{r}, r=1,2,3$. Оценка, полученная для $\zeta_{3}$, оптимальна. Кроме того, доказано, что константа $C$, фигурирующая в неравенстве Берри-Эссеена, не превосходит 0.5894. Удалось также оценить соответствующую константу, возникающую в аналогичном неравенстве для неодинаково распределенных слагаемых.
\end{abstract}

Ключевые слова и фразы: центральная предельная теорема, скорость сходимости, неравенство Берри-Эссеена, нулевое смещение.

1. Введение. Рассмотрим независимые (действительные) случайные величины (с.в.) $X_{1}, \ldots, X_{n}$ с нулевыми средними, дисперсиями равными $\sigma_{1}^{2}, \ldots, \sigma_{n}^{2}$ и конечными третьими абсолютными моментами $\beta_{1}, \ldots, \beta_{n}$. Обозначим

$$
\sigma^{2}=\sigma^{2}(n):=\sum_{j=1}^{n} \sigma_{j}^{2}, \quad \varepsilon_{n}:=\frac{1}{\sigma^{3}} \sum_{j=1}^{n} \beta_{j}
$$

Согласно теореме Ляпунова $S_{n}:=\left(X_{1}+\cdots+X_{n}\right) / \sigma(n)$ слабо сходится к стандартной нормальной с.в. при $\varepsilon_{n} \rightarrow 0$. С теоретической и практической точек зрения немалый интерес представляет оценка скорости сходимости в этой теореме. Как известно (см. [1], [2]), существует такая наименьшая постоянная $C$, что для расстояния Колмогорова между $S_{n}$ и стандартной гауссовской величиной $N$ справедливо неравенство

$$
\rho\left(S_{n}, N\right):=\sup _{x \in \mathbf{R}}\left|\mathbf{P}\left(S_{n} \leqslant x\right)-\mathbf{P}(N \leqslant x)\right| \leqslant C \varepsilon_{n}, \quad n \in \mathbf{N} .
$$

* Московский государственный университет им. М.В.Ломоносова, кафедра теории вероятностей, Ленинские горы, 119991 Москва, ГСП-1, Россия; е-mail: itiurin@gmail.com 
Оценке этой константы посвящено множество работ. Эссеен [2] показал, что $C \leqslant 7.5$. Бергстрем [3] получил оценку $C \leqslant 4.8$. Такано [4] установил, что в случае независимых одинаково распределенных (н.о.р.) слагаемых $C \leqslant 2.031$. В. М. Золотарев (см. [5]-[8]) получил новое неравенство, позволяющее оценивать близость двух сумм независимых с.в. и с его помощью показал последовательно справедливость оценок $C \leqslant 1.322$ и $C \leqslant 0.9051$, а для случая н.о.p. величин $C \leqslant 1.301$ и $C \leqslant 0.8197$. Предложенный метод получил развитие в работах ван Бика [9] и И. С. Шиганова [10], которые доказали соответственно оценки $C \leqslant 0.7975$ и $C \leqslant 0.7915$. Для сумм с.в., имеющих одинаковое распределение, И. С. Шиганов получил оценку $C \leqslant 0.7655$, которую в 2006 году улучшила И. Г. Шевцова [11], показав, что в этом случае $C \leqslant 0.7056$.

Отдельного упоминания заслуживает родственная задача отыскания асимптотически правильных постоянных в теореме Ляпунова. Как показал Эссеен [12], если все с.в. $X_{j}, j=1,2, \ldots$, имеют одно и то же распределение, то

$$
\limsup _{n \rightarrow \infty} \frac{\rho\left(S_{n}, N\right)}{\varepsilon_{n}} \leqslant C_{1}:=\frac{\sqrt{10}+3}{6 \sqrt{2 \pi}}=0.409 \ldots
$$

причем константа в правой части этого неравенства не может быть уменьшена (откуда, в частности, следует нижняя оценка $C \geqslant C_{1}$ ). Последний результат был развит Б. А. Рогозиным [13], который установил, что при тех же предположениях

$$
\limsup _{n \rightarrow \infty} \frac{\rho\left(S_{n}, \mathscr{N}\right)}{\varepsilon_{n}} \leqslant C_{2}:=\frac{1}{\sqrt{2 \pi}},
$$

где $\rho\left(S_{n}, \mathscr{N}\right):=\inf _{G \in \mathscr{N}} \rho\left(S_{n}, G\right), \quad \mathscr{N}-$ множество всех нормальных с.в.

Г. П. Чистяков (см. [14]-[16]) обобщил (2) и (3) на случай неодинаково распределенных слагаемых. Он доказал, что

$$
\rho\left(S_{n}, N\right) \leqslant C_{1} \varepsilon_{n}+r_{1}\left(\varepsilon_{n}\right), \quad \rho\left(S_{n}, \mathscr{N}\right) \leqslant C_{2} \varepsilon_{n}+r_{2}\left(\varepsilon_{n}\right),
$$

где $r_{1}\left(\varepsilon_{n}\right), r_{2}\left(\varepsilon_{n}\right)$ есть $o\left(\varepsilon_{n}\right)$ при $\varepsilon_{n} \rightarrow 0$.

Известны также оценки скорости сходимости в теореме Ляпунова при наличии моментов порядка $2+\delta$ (см. [17], [18]).

Аналоги (1) существуют и для других вероятностных метрик, например $\zeta_{r}$ (где $r=1,2,3$ ), о которых подробнее будет сказано в п. 2. Оценки в этих метриках могут быть получены естественным образом при использовании метода Стейна. При доказательстве упомянутых оценок, в частности, используется так называемое нулевое смещение вероятностного распределения (см. [19]). 
Для расстояния в метриках $\zeta_{r}(r=1,2,3)$ известны следующие оценки (см. [20]):

$$
\zeta_{1}\left(S_{n}, N\right) \leqslant 3 \varepsilon_{n}, \quad \zeta_{2}\left(S_{n}, N\right) \leqslant \frac{3 \sqrt{2 \pi}}{8} \varepsilon_{n}, \quad \zeta_{3}\left(S_{n}, N\right) \leqslant \frac{1}{2} \varepsilon_{n} .
$$

В [21] рассмотрена задача об отыскании наименьшей верхней грани $\mathbf{E} f\left(X_{1}, \ldots, X_{n}\right)$ по множеству наборов независимых простых с.в., удовлетворяющих $m$ ограничениям вида $\mathbf{E} g_{i j}\left(X_{j}\right)=c_{i j}, j=1, \ldots, n$. А именно, установлено, что в этом случае можно ограничиться рассмотрением с.в., принимающих не более $m+1$ значений. В настоящей работе результаты [21] обобщены на случай произвольного квазивыпуклого функционала, заданного на множестве вероятностных распределений.

При помощи полученных результатов установлена неулучшаемая оценка близости в средней метрике вероятностного распределения и его преобразования нулевого смещения. Последнее применено к оценке точности гауссовской аппроксимации распределений нормированных сумм независимых слагаемых. Установлено, что значения констант в (4) можно уменьшить в 3 раза. При этом, наша оценка, полученная для метрики $\zeta_{3}$, оптимальна. Кроме того, выведены новые оценки близости характеристических функций нормированной суммы и стандартной нормальной с.в. Это позволило доказать, что $C \leqslant 0.6379$, а в случае н.о.р. слагаемых $C \leqslant 0.5894$.

2. Обозначения и формулировки результатов. Пусть $(S, d)-$ некоторое метрическое пространство, $Q$ - множество зарядов ограниченной вариации на $\sigma$-алгебре $\mathscr{B}(S)$ борелевских подмножеств $S$ с операциями умножения на скаляр и сложения, определенными следующим образом: пусть $\mu, \mu_{1}, \mu_{2} \in Q, c \in \mathbf{R}$, положим для каждого $A \in \mathscr{B}(S)$

$$
(c \mu)(A):=c \cdot \mu(A), \quad\left(\mu_{1}+\mu_{2}\right)(A):=\mu_{1}(A)+\mu_{2}(A) .
$$

Легко видеть, что $Q$ замкнуто относительно этих операций и представляет собой линейное пространство, а множество $D$ дискретных вероятностных мер, сосредоточенных в конечном числе точек, есть выпуклое подмножество $Q$. Последнее означает, что $\alpha \mu_{1}+(1-\alpha) \mu_{2} \in D$ для произвольных $\mu_{1}, \mu_{2} \in D$ и $\alpha \in(0,1)$.

Рассмотрим множество наборов, состоящих из $n$ независимых с.в. $X_{1}, \ldots, X_{n}$. Тогда

$$
\mathbf{E} f\left(X_{1}, \ldots, X_{n}\right)=\int_{\mathbf{R}^{n}} f d P_{X_{1}} \ldots d P_{X_{n}},
$$

где $f: \mathbf{R}^{n} \rightarrow \mathbf{R}, P_{X_{1}}, \ldots, P_{X_{n}}-$ распределения с.в. $X_{1}, \ldots, X_{n}$. Таким образом, $\mathbf{E} f\left(X_{1}, \ldots, X_{n}\right)$ можно рассматривать как функцию на множестве мер, линейную по каждому из $n$ своих аргументов. 
Функция $g: G \rightarrow \mathbf{R}$, где $G-$ выпуклое множество, называется квазивыпуклой, если для любых $x, y \in G$ и $\alpha \in(0,1)$ имеем

$$
g(\alpha x+(1-\alpha) y) \leqslant \max \{g(x), g(y)\} .
$$

Всюду далее будем предполагать, что заданы $h_{1}, \ldots, h_{m}$ - некоторые вещественнозначные функции на $S$. Рассмотрим множество

$$
K:=\left\{\mu \in D:\left\langle h_{i}, \mu\right\rangle=0, i=1, \ldots, m\right\}, \quad \text { где }\langle f, \mu\rangle:=\int_{S} f d \mu .
$$

Нетрудно видеть, что $K-$ выпуклое множество. Кроме того, обозначим $K_{j}$ - множество мер $\mu \in K$, сосредоточенных на не более чем $j$ точках $(j \in \mathbf{N})$.

Теорема 1. Для произвольной квазивыпуклой функции $g: K \rightarrow \mathbf{R}$ имеем

$$
\sup _{\mu \in K} g(\mu)=\sup _{\mu \in K_{m+1}} g(\mu) .
$$

В последнем выражении супремум по пустому множеству считаем равным нулю.

Теорема 2. Пусть $f$ - неотрицательная функиия на $S, V-$ векторное пространство с нормой $\|\cdot\|, A: K \rightarrow V$ - такое отображение, umo

$$
A(\alpha \mu+(1-\alpha) \nu)=\alpha A \mu+(1-\alpha) A \nu
$$

для любых $\mu, \nu \in K, \alpha \in(0,1)$. Тогда наименьшее $\gamma$, при котором неравенство

$$
\|A \mu\| \leqslant \gamma\langle f, \mu\rangle
$$

выполнено для любой меры $\mu \in K$, совпадает с наименьшим $\gamma$, при котором (6) верно для произвольной меры $\mu \in K_{m+1}$.

Пусть $W-$ с.в. с нулевым средним и дисперсией $\sigma^{2}>0$. Говорят, что с.в. $W^{*}$ имеет распределение $W$-нулевого смещения, если

$$
\mathbf{E} W f(W)=\sigma^{2} \mathbf{E} f^{\prime}\left(W^{*}\right)
$$

для каждой дифференцируемой функции $f: \mathbf{R} \rightarrow \mathbf{R}$, для которой левая часть (7) определена. Известно (см. [19]), что $W^{*}$ существует для любой описанной выше $W$ и имеет плотность

$$
p(w)= \begin{cases}\sigma^{-2} \mathbf{E}(W \cdot \mathbf{1}\{W>w\}), & \text { если } w \geqslant 0 ; \\ \sigma^{-2} \mathbf{E}(-W \cdot \mathbf{1}\{W<w\}), & \text { если } w<0 .\end{cases}
$$

Для произвольной функции $f \in C^{(r-1)}(\mathbf{R})$, где $r \in \mathbf{N}$, положим

$$
M_{r}(f):=\sup _{x \neq y}\left|\frac{f^{(r-1)}(x)-f^{(r-1)}(y)}{x-y}\right| .
$$


Как обычно, $C^{(0)}(\mathbf{R}):=C(\mathbf{R})$. Если $f \notin C^{(r-1)}(\mathbf{R})$, то считаем $M_{r}(f)=$ $\infty$. Положим

$$
\zeta_{r}(X, Y):=\sup \left\{|\mathbf{E} f(X)-\mathbf{E} f(Y)|: f \in \mathscr{F}_{r}\right\}, \quad r=1,2, \ldots,
$$

где $\mathscr{F}_{r}$ - множество вещественных ограниченных функций с $M_{r}(f) \leqslant 1$.

Заметим, что $\zeta_{1}$ имеет другие представления. Это так называемая средняя метрика

$$
\varkappa_{1}(X, Y):=\int_{-\infty}^{\infty}|\mathbf{P}(X \leqslant x)-\mathbf{P}(Y \leqslant x)| d x
$$

а также минимальная $L_{1}$-метрика

$$
l_{1}(X, Y):=\inf \{\mathbf{E}|\tilde{X}-\tilde{Y}|: \operatorname{Law}(\tilde{X})=\operatorname{Law}(X), \operatorname{Law}(\tilde{Y})=\operatorname{Law}(Y)\} .
$$

По этому поводу см. [22, с. 56].

Теорема 3. Пусть $W$ чентрирована, имеет единичную дисперсию и конечньй третий абсолютньй момент. Тогда

$$
\zeta_{1}\left(W, W^{*}\right) \leqslant \frac{1}{2} \mathbf{E}|W|^{3},
$$

причем равенство достигается, когда $W$ принимает ровно два значения.

Следствие 1. Пусть с.в. $S_{n}^{*}$ имеет распределение $S_{n}$-нулевого смещения. Тогда

$$
\zeta_{1}\left(S_{n}, S_{n}^{*}\right) \leqslant \frac{1}{2} \varepsilon_{n}
$$

Теорема 4. Справедливь следующие неравенства:

$$
\begin{gathered}
\zeta_{1}\left(S_{n}, N\right) \leqslant 2 \zeta_{1}\left(S_{n}, S_{n}^{*}\right) \leqslant \varepsilon_{n}, \quad \zeta_{2}\left(S_{n}, N\right) \leqslant \frac{\sqrt{2 \pi}}{4} \zeta_{1}\left(S_{n}, S_{n}^{*}\right) \leqslant \frac{\sqrt{2 \pi}}{8} \varepsilon_{n}, \\
\zeta_{3}\left(S_{n}, N\right) \leqslant \frac{1}{3} \zeta_{1}\left(S_{n}, S_{n}^{*}\right) \leqslant \frac{1}{6} \varepsilon_{n} .
\end{gathered}
$$

Последнее двойное неравенство оптимально, а именно, для любого $\delta>0$ существует такая последовательность н.о.р. с.в. $X_{1}, X_{2}, \ldots$, umo

$$
\frac{\zeta_{3}\left(S_{n}, N\right)}{\varepsilon_{n}} \geqslant \frac{1}{6}-\delta, \quad n=1,2, \ldots
$$

Для $\gamma>0$ и $t \in \mathbf{R}$ положим

$$
b(t, \gamma):= \begin{cases}-t^{2}+2 \gamma a|t|^{3}, & \text { если } \gamma|t|<M \\ -2\left(\frac{1}{\gamma}\right)^{2}(1-\cos \gamma t), & \text { если } M \leqslant \gamma|t| \leqslant 2 \pi \\ 0, & \text { если } \gamma|t|>2 \pi .\end{cases}
$$


Здесь $a:=\max _{x>0}\left\{\left(\cos (x)-1+x^{2} / 2\right) / x^{3}\right\} \approx 0.099162, M$ - точка, в которой достигается этот максимум, $M \approx 3.995896$.

Обозначим $f_{S_{n}}(t):=\mathbf{E} e^{i t S_{n}}, \varphi(t):=\exp \left(-t^{2} / 2\right), \delta_{n}(t):=\mid f_{S_{n}}(t)-$ $\varphi(t) \mid, t \in \mathbf{R}$.

Теорема 5. Для произвольного $t \in \mathbf{R}$ имеем

$$
\begin{aligned}
& \delta_{n}(t) \leqslant \delta^{(1)}\left(\varepsilon_{n}, t\right):=\exp \left(\frac{1}{2} b\left(t, 2 \varepsilon_{n}\right)\right)+\varphi(t), \\
& \delta_{n}(t) \leqslant \delta^{(2)}\left(\varepsilon_{n}, t\right):=\varepsilon_{n} \varphi(t) \int_{0}^{|t|} \frac{s^{2}}{2} \exp \left(\frac{s^{2}}{2}\right) d s .
\end{aligned}
$$

Обозначим $A:=\varepsilon_{n}^{-1 / 3} / 6$. Для всех $t \in \mathbf{R}$ верна оченка

$$
\begin{aligned}
& \delta_{n}(t) \leqslant \delta^{(3)}\left(\varepsilon_{n}, t\right) \\
& =\left\{\begin{array}{l}
\varepsilon_{n} \varphi(t) \int_{0}^{|t|}\left(s^{2} / 2\right) \exp \left(s^{2} \varepsilon_{n}^{2 / 3} / 2\right) d s, \quad|t| \leqslant A \\
\varepsilon_{n} \varphi(t)\left(\int_{0}^{A}\left(s^{2} / 2\right) \exp \left(s^{2} \varepsilon_{n}^{2 / 3} / 2\right) d s+\int_{A}^{|t|}\left(s^{2} / 2 l\right) \exp \left(2 a \varepsilon_{n} s^{3}\right) d s\right), \\
|t|>A .
\end{array}\right.
\end{aligned}
$$

әде

$$
\left.l:=\inf _{t \geqslant 0}\left\{\exp \left(-t^{2} / 2+2 a t^{3}\right)\right)\right\} \approx 0.624489 .
$$

Для случая н.о.р. величин оценки могут быть несколько улучшены. Обозначим $\tau_{n}:=\sigma^{-3} \sum_{j=1}^{n} \sigma_{j}^{3}$. Пусть $X_{1}, X_{2}, \ldots$ - центрированные н.о.р. с.в. с дисперсиями 1 и конечными третьими абсолютными моментами $\beta$. Тогда $\varepsilon_{n}=\beta / \sqrt{n}, \tau_{n}=1 / \sqrt{n}$.

Теорема 6. Для описанной последовательности с.в. и произвольного $t \in \mathbf{R}$ справедливь неравенства

$$
\begin{aligned}
\delta_{n}(t) & \leqslant \delta^{(4)}\left(n, \varepsilon_{n}, t\right):=\left(1+\frac{b\left(t, \varepsilon_{n}+\tau_{n}\right)}{n}\right)^{n / 2}+\varphi(t), \\
\delta_{n}(t) & \leqslant \delta^{(5)}\left(n, \varepsilon_{n}, t\right) \\
& :=\varepsilon_{n} \varphi(t) \int_{0}^{|t|}\left(1+\frac{b\left(s, \varepsilon_{n}+\tau_{n}\right)}{n}\right)^{(n-1) / 2} \frac{s^{2}}{2} \exp \left(\frac{s^{2}}{2}\right) d s .
\end{aligned}
$$

Пусть $m \in \mathbf{N} u n \geqslant m$. Тогда

$$
\begin{aligned}
\delta_{n}(t) & \leqslant \delta^{(6)}\left(m, \varepsilon_{n}, t\right):=\exp \left(\frac{1}{2} b\left(t, \varepsilon_{n}+\tau_{m}\right)\right)+\varphi(t) \\
\delta_{n}(t) & \leqslant \delta^{(7)}\left(m, \varepsilon_{n}, t\right) \\
& :=\varepsilon_{n} \varphi(t) \int_{0}^{|t|} \exp \left(\frac{m-1}{2 m} b\left(s, \varepsilon_{n}+\tau_{m}\right)+\frac{s^{2}}{2}\right) \frac{s^{2}}{2} d s .
\end{aligned}
$$


Оценки (11)-(17) позволили установить следующий результат.

Теорема 7. Неравенство (1) имеет место с константой $C \leqslant$ 0.6379, а в случае одинаково распределенных слагаемых $C \leqslant 0.5894$.

\section{3. Доказательства основных результатов.}

Доказ а т ель с т в о т е о ре мы 1 . Если $K=\varnothing$, то $K_{m+1}=$ $\varnothing$, и утверждение теоремы выполнено. Далее предполагаем, что множество $K$ непусто.

Последовательность множеств $K_{1}, K_{2}, \ldots$ возрастает к множеству $K$, следовательно,

$$
\sup _{\mu \in K} g(\mu)=\sup _{j \geqslant 1} \sup _{\mu \in K_{j}} g(\mu)=\sup _{j \geqslant m+1} \sup _{\mu \in K_{j}} g(\mu) .
$$

Поэтому достаточно установить, что

$$
\sup _{\mu \in K_{m+1}} g(\mu) \geqslant \sup _{\mu \in K_{m+2}} g(\mu) \geqslant \sup _{\mu \in K_{m+3}} g(\mu) \geqslant \cdots .
$$

Для этого возьмем произвольную меру $\mu \in K_{j}$, где $j>m+1$, и покажем, что существует $\mu^{\prime} \in K_{j-1}$, такая, что $g\left(\mu^{\prime}\right) \geqslant g(\mu)$.

Пусть $\mu$ сосредоточена в точках $s_{1}, \ldots, s_{j} \in S$ и

$$
\mu\left(s_{i}\right)=\mu_{i} \geqslant 0, \quad i=1, \ldots, j .
$$

Вектор $\bar{\mu}=\left(\mu_{1}, \ldots, \mu_{j}\right)$ задает вероятностное распределение, поэтому

$$
\mu_{1}+\cdots+\mu_{j}=1 .
$$

Кроме того, выполнены условия $\left\langle h_{i}, \mu\right\rangle=0, i=1, \ldots, m$, и, следовательно,

$$
\mu_{1} \cdot h_{i}\left(s_{1}\right)+\cdots+\mu_{j} \cdot h_{i}\left(s_{j}\right)=0, \quad i=1, \ldots, m .
$$

Обратно, всякий вектор с неотрицательными координатами, удовлетворяющий системе линейных уравнений (19) и (20) задает согласно формулам (18) элемент множества $K_{j}$, а если одна из его координат равна нулю, то элемент $K_{j-1}$. Имеем $m+1$ уравнение и как минимум $m+2$ неизвестных, следовательно, существует ненулевое решение $\bar{\nu}=\left(\nu_{1}, \ldots, \nu_{j}\right)$ соответствующей однородной системы. Поскольку сумма координат этого вектора равна нулю, но сам вектор ненулевой, то у него есть положительная и отрицательная координаты. Поэтому существуют такие наименьшие $\alpha \geqslant 0$ и $\beta \geqslant 0$, что соответственно одна из координат вектора $\overline{\mu_{*}}=\bar{\mu}-\alpha \bar{\nu}$ равна нулю, и некоторая координата $\overline{\mu^{*}}=\bar{\mu}+\beta \bar{\nu}$ равна нулю. Если $\alpha=0$, то $\mu \in K_{j-1}$, иначе

$$
\bar{\mu}=\frac{\beta}{\alpha+\beta} \bar{\mu}_{*}+\frac{\alpha}{\alpha+\beta} \bar{\mu}^{*},
$$


и в силу квазивыпуклости $g(\mu) \leqslant \max \left\{g\left(\mu_{*}\right), g\left(\mu^{*}\right)\right\}$, где $\mu_{*}, \mu^{*}-$ распределения, отвечающие $\bar{\mu}_{*}$ и $\bar{\mu}^{*}$. Таким образом $g(\mu) \leqslant g\left(\mu_{*}\right)$ либо $g(\mu) \leqslant g\left(\mu^{*}\right)$. Но $\mu_{*}$ и $\mu^{*} \in K_{j-1}$.

Д о к а з а т е л ь с т в о т е о р е м ы 2. В силу теоремы 1 достаточно показать, что при каждом фиксированном значении $\gamma$ функция $g(\mu):=\|A \mu\|-\gamma\langle f, \mu\rangle$ является квазивыпуклой. Пусть $\alpha+\beta=1$. Согласно свойствам нормы

$$
\begin{aligned}
\|A(\alpha \mu+\beta \nu)\|-\gamma\langle f, \alpha \mu+\beta \nu\rangle & =\|\alpha A \mu+\beta A \nu\|-\gamma\langle f, \alpha \mu\rangle-\gamma\langle f, \beta \nu\rangle \\
& \leqslant\|\alpha A \mu\|+\|\beta A \nu\|-\gamma\langle f, \alpha \mu\rangle-\gamma\langle f, \beta \nu\rangle \\
& =\alpha g(\mu)+\beta g(\nu) \leqslant \max \{g(\mu), g(\nu)\} .
\end{aligned}
$$

Теорема 2 доказана.

Д ок аз а т е л с с в о т е о р е мы 3 . Вначале проверим, что можно ограничиться рассмотрением простых с.в. $W$. Достаточно показать, что для любой с.в. $W$, удовлетворяющей условиям теоремы, существует такая последовательность $\left(W_{n}\right)_{n \geqslant 1}$ простых с.в. с нулевым средним и единичной дисперсией, такая, что

$$
\zeta_{1}\left(W_{n}, W_{n}^{*}\right) \rightarrow \zeta_{1}\left(W, W^{*}\right) \quad \text { и } \quad \mathbf{E}\left|W_{n}\right|^{3} \rightarrow \mathbf{E}|W|^{3}, \quad n \rightarrow \infty .
$$

Считаем, что случайная величина $W$ задана на вероятностном пространстве $\left(\mathbf{R}, \mathscr{B}(\mathbf{R}), \mathbf{P}=P_{W}\right)$. Построим последовательность простых с.в. $\left(W_{n}^{\prime}\right)_{n \geqslant 1}$, сходяшуюся к $W$ по норме $L_{3}$. Положим

$$
W_{n}:=\frac{W_{n}^{\prime}-\mathbf{E} W_{n}^{\prime}}{\sqrt{\operatorname{Var} W_{n}^{\prime}}}
$$

Легко видеть, что $W_{n}$ тоже сходится к $W$ по норме $L_{3}$. Поэтому второе из условий (21), очевидно, выполнено. Остается показать справедливость первого из этих условий.

Из неравенства треугольника для метрики $\zeta_{1}$ нетрудно получить

$$
\left|\zeta_{1}\left(W, W^{*}\right)-\zeta_{1}\left(W_{n}, W_{n}^{*}\right)\right| \leqslant \zeta_{1}\left(W, W_{n}\right)+\zeta_{1}\left(W^{*}, W_{n}^{*}\right) .
$$

Первое слагаемое в правой части (22) стремится к нулю, поскольку

$$
\zeta_{1}\left(W, W_{n}\right)=l_{1}\left(W, W_{n}\right) \leqslant \mathbf{E}\left|W-W_{n}\right| \leqslant\left(\mathbf{E}\left|W-W_{n}\right|^{3}\right)^{1 / 3} .
$$

Оценим второе слагаемое. Для функции $f \in \mathscr{F}_{1}$ положим $F(x):=$ $\int_{0}^{x} f(u) d u$. Тогда

$$
\mathbf{E} f\left(W^{*}\right)-\mathbf{E} f\left(W_{n}^{*}\right)=\mathbf{E} W F(W)-\mathbf{E} W_{n} F\left(W_{n}\right) .
$$

Поскольку при замене функции $f(x)$ на $f(x)-f(0)$ разность математических ожиданий в левой части $(23)$ не изменится, можем считать без 
ограничения общности, что $f(0)=0$. Тогда $|f(x)-f(y)| \leqslant|x-y|$ влечет $|f(x)| \leqslant|x|$ и, как следствие, $|F(x)| \leqslant|x|^{2},|x f(x)| \leqslant|x|^{2}$. По теореме о конечном приращении

$W F(W)-W_{n} F\left(W_{n}\right)=\left.\left(W-W_{n}\right) \cdot\{x F(x)\}^{\prime}\right|_{x=\xi}=\left(W-W_{n}\right)\{F(\xi)+\xi f(\xi)\}$, где $\xi$ - число между $W$ и $W_{n}$. Последнее, с учетом того, что

$$
|F(\xi)+\xi f(\xi)| \leqslant 2|\xi|^{2} \leqslant 2\left(|W|+\left|W_{n}\right|\right)^{2},
$$

дает оценку

$$
\left|\mathbf{E}\left\{W F(W)-W_{n} F\left(W_{n}\right)\right\}\right| \leqslant 2 \mathbf{E}\left|W-W_{n}\right|\left(|W|+\left|W_{n}\right|\right)^{2} .
$$

Наконец, из неравенства Гельдера

$$
\mathbf{E}\left|W-W_{n}\right|\left(|W|+\left|W_{n}\right|\right)^{2} \leqslant\left(\mathbf{E}\left|W-W_{n}\right|^{3}\right)^{1 / 3}\left(\mathbf{E}\left(|W|+\left|W_{n}\right|\right)^{3}\right)^{2 / 3} .
$$

Таким образом к нулю стремится и второе слагаемое (22), поскольку $\left(\mathbf{E}\left|W-W_{n}\right|^{3}\right)^{1 / 3} \rightarrow 0$ по определению сходимости в $L_{3}$.

Итак, достаточно рассматривать только простые с.в. Пусть $A_{1}-$ отображение, сопоставляющее распределению $P_{X}$ с.в. $X$ распределение ее нулевого смещения $P_{X}^{*}$. Кроме того, рассмотрим линейный оператор $A_{2}$, сопоставляющий заряду $\nu$ его функцию распределения (ф.р.) $G_{\nu}(x):=\nu((-\infty, x])$. Нетрудно видеть, что

$$
\left(\alpha P_{W_{1}}+(1-\alpha) P_{W_{2}}\right)^{*}=\alpha P_{W_{1}}^{*}+(1-\alpha) P_{W_{2}}^{*},
$$

поэтому отображение $A_{2}-A_{2} A_{1}$ удовлетворяет (5). Если положить $h_{1}(x)=x, h_{2}(x)=x^{2}-1$ и в теореме 2 взять $f(x)=|x|^{3}, A=A_{2}-A_{2} A_{1}$, $V$ - пространство интегрируемых (по мере Лебега) на вещественной прямой функций с нормой

$$
\|G\|=\int_{-\infty}^{\infty}|G(x)| d x
$$

то получим, что достаточно рассматривать только простые с.в., принимающие не более трех значений. Функция распределения простой с.в. $W$ имеет ступенчатый вид. По формуле (8) легко получить фунцкию распределения $W^{*}$. Зная обе эти функции, не составляет труда найти явное выражение расстояния в средней метрике $\varkappa_{1}\left(W, W^{*}\right)$.

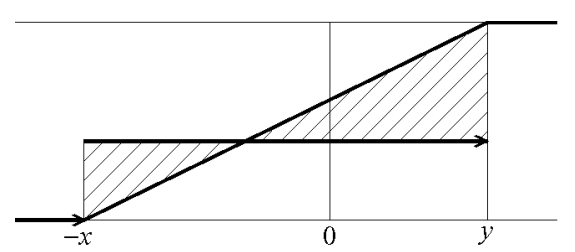

a)

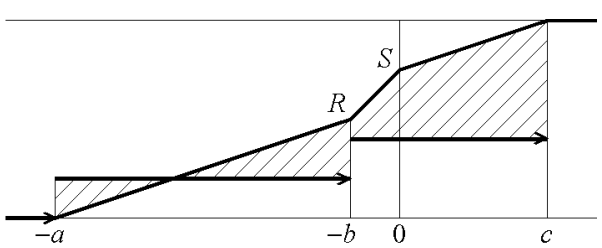

6)

Рис. 1 
Пусть $W$ принимает ровно два значения $-x$ и $y$ с вероятностями $p$ и $q$. Тогда ее функция распределения кусочно-постоянна и имеет два скачка в точках $-x$ и $y$ равных соответственно $p$ и $q$. Так как $W$ центрирована, имеем $p x=q y$. Откуда, согласно $(8)$, следует, что $W^{*}$ распределена равномерно на отрезке $[-x, y]$. Следовательно, на $[-x, y]$ ее функция распределения линейна, а график представляет собой отрезок, соединяющий точки $(-x, 0)$ и $(y, 1)$. По определению $\varkappa_{1}\left(W, W^{*}\right)$ равна площади фигуры, заключенной между функцией распределения (в рассматриваемом случае это объединение двух треугольников, см. рис. 1 а)).

Из того, что $\mathbf{E} W=0$ и $\mathbf{E} W^{2}=1$ следует, что $x=\sqrt{q / p}, y=\sqrt{p / q}$. Отсюда

$$
\mathbf{E}|W|^{3}=p x^{3}+q y^{3}=q \sqrt{\frac{q}{p}}+p \sqrt{\frac{p}{q}} .
$$

Найдем площадь фигуры, заключенной между функцией распределения с.в. $W$ и $W^{*}$. Плотность $W^{*}$ равна, $p x=q y=\sqrt{p q}$. Следовательно, функция распределения этой с.в. на отрезке $[-x, y]$ имеет коэффициент наклона $\sqrt{p q}$. Длина вертикального катета первого треугольника равна $p$, а второго $q$. Отсюда сумма площадей этих треугольников равна

$$
\frac{1}{2} p^{2} \frac{1}{\sqrt{p q}}+\frac{1}{2} q^{2} \frac{1}{\sqrt{p q}}=\frac{1}{2} p \sqrt{\frac{p}{q}}+\frac{1}{2} q \sqrt{\frac{q}{p}}=\frac{1}{2} \mathbf{E}|W|^{3} .
$$

Итак, если $W$ принимает ровно два значения, в (9) имеем равенство.

Рассмотрим случай, когда $W$ принимает три значения. Считаем без ограничения общности, что два из них $(-a<-b)$ не превосходят нуля, а одно $(c)$ положительно. Как и ранее функция распределения с.в. $W$ кусочно постоянна, а функция распределения $W^{*}$ кусочно-линейна, однако форма фигуры, заключенной между ними, имеет более сложный вид (см. рис. 1б)). Обозначим $R$ значение функции распределения с.в. $W^{*}$ в точке $-b, S-$ ее значение в точке 0 . Пусть $W$ принимает значения $-a$, $-b, c$ соответственно с вероятностями $p, q, r$. Тогда в силу моментных ограничений

$$
\left\{\begin{array}{l}
p+q+r=1, \\
-p a-q b+r c=0, \\
p a^{2}+q b^{2}+r c^{2}=1
\end{array}\right.
$$

Это система линейных уравнений относительно $p, q, r$. Воспользовавшись правилом Крамера, получим

$$
p=(1-b c)(c+b) / \Delta, \quad q=(a c-1)(a+c) / \Delta, \quad r=(a b+1)(a-b) / \Delta,
$$

где $\Delta=(a+c)(b+c)(a-b)$. Таким образом, всякая с.в. с нулевым средним и дисперсией 1 , принимающая три значения, определяется этими тремя 
значениями однозначно. Легко видеть, что $p, q, r$ неотрицательны тогда и только тогда, когда

$$
a c \geqslant 1, \quad b c \leqslant 1 .
$$

Другими словами, с.в. $W$, принимающая значения $-a,-b, c$ существует в том и только том случае, когда выполнено (25). Наша цель - доказать, что функция

$$
g(a, b, c):=\varkappa_{1}\left(W, W^{*}\right)-\frac{1}{2} \mathbf{E}|W|^{3}
$$

не превосходит нуля. Ее явное выражение через переменные $a, b, c$ зависит от взаимного расположения функций распределения с.в. $W$ и $W^{*}$. Имеется 5 случаев:

I. $R \leqslant p, S \leqslant p+q$, или, эквивалентно, $a(a-b) \leqslant 1, c \geqslant 1$. В этом случае

$$
g(a, b, c)=\frac{r}{c}-p a^{3}-q b^{3} .
$$

II. $R \leqslant p, S \geqslant p+q \Leftrightarrow a(a-b) \leqslant 1, c \leqslant 1$,

$$
g(a, b, c)=\frac{r}{c}-p a^{3}-q b^{3} .
$$

III. $R \geqslant p, S \leqslant p+q \Leftrightarrow a(a-b) \geqslant 1, c \geqslant 1$ (последнее влечет $c(b+c) \geqslant 1)$,

$$
g(a, b, c)=p a\left\{a-b-\frac{1}{a}\right\}^{2}+\frac{r}{c}-p a^{3}-q b^{3} .
$$

IV. $p \leqslant R \leqslant p+q, S \geqslant p+q \Leftrightarrow a(a-b) \geqslant 1, c(b+c) \geqslant 1, c \leqslant 1$,

$$
g(a, b, c)=p a\left\{a-b-\frac{1}{a}\right\}^{2}+\frac{r}{c}-p a^{3}-q b^{3} .
$$

V. $R \geqslant p+q \Leftrightarrow c(b+c) \leqslant 1$,

$$
g(a, b, c)=\frac{p}{a}-r c^{3} .
$$

Заметим, что в каждом из случаев $g$ - это одна и та же функция, заданная соотношением (26). Поэтому, если набор значений $a, b, c$ удовлетворяет ограничениям сразу двух случаев, для функции $g$ можно использовать как выражение, соответствующее первому из них, так и выражение, соответствующее второму.

Как видно, в случаях I, II, а также в случаях III, IV функция $g$ имеет одно и то же представление. I и II объединим в случай A, III и IV - в случай В.

A. $a(a-b) \leqslant 1$,

$$
g(a, b, c)=\frac{r}{c}-p a^{3}-q b^{3} .
$$


B. $a(a-b) \geqslant 1, c(b+c) \geqslant 1$,

$$
g(a, b, c)=p a\left\{a-b-\frac{1}{a}\right\}^{2}+\frac{r}{c}-p a^{3}-q b^{3} .
$$

Покажем, что в каждом из случаев $\mathrm{A}, \mathrm{B}, \mathrm{V}$ функция $g$ не превосходит нуля.

Случай A.

$$
\begin{aligned}
g(a, b, c) & =\frac{r}{c}-p a^{3}-q b^{3} \\
& =\frac{(1+a b)(a-b)}{\Delta c}-\frac{(1-b c)(b+c) a^{3}}{\Delta}-\frac{(a c-1)(a+c) b^{3}}{\Delta} \\
& =\frac{(a-b)(a c-1)(1-b c)}{\Delta c}(-1-b c-a c-a b) \leqslant 0
\end{aligned}
$$

в силу (25), неотрицательности чисел $a, b, c$ и того, что $a>b$.

Случай V.

$$
\begin{aligned}
g(a, b, c) & =\frac{p}{a}-r c^{3}=\frac{(1-b c)(b+c)}{\Delta a}-\frac{(1+a b)(a-b) c^{3}}{\Delta} \\
& =\frac{(a c-1)}{\Delta a}\left\{-[(a b+1)(a-b)] c^{2}-[1+b(a-b)] c-b\right\} .
\end{aligned}
$$

Поскольку $a c \geqslant 1$ достаточно доказать, что выражение, стоящее внутри фигурных скобок, не превосходит нуля. Рассмотрим это выражение как функцию от переменной $c$, зафиксировав остальные. При $c=0$ эта функция равна $-b \leqslant 0$. Осталось заметить, что она убывает по $c$, поскольку коэффициенты при $c$ и $c^{2}$ отрицательны, и, как следствие, не превосходит нуля при любых положительных значениях $c$.

Случай В.

$$
\begin{aligned}
g(a, b, c)= & p a\left\{a-b-\frac{1}{a}\right\}^{2}+\frac{r}{c}-p a^{3}-q b^{3} \\
= & \frac{(1-b c)(b+c) a}{\Delta}\left\{a-b-\frac{1}{a}\right\}^{2} \\
& +\frac{(1+a b)(a-b)}{\Delta c}-\frac{(1-b c)(b+c) a^{3}}{\Delta}-\frac{(a c-1)(a+c) b^{3}}{\Delta} \\
= & \frac{1-b c}{\Delta a c}\left\{k_{2} c^{2}+k_{1} c+k_{0}\right\}
\end{aligned}
$$

где $k_{2}=1-2 a(a-b)(1+a b), k_{1}=b[1-a(a-b)(1+a b)], k_{0}=a(a-b)(1+a b)$. Допустим, что $g(a, b, c)>0$. В силу условия $a(a-b) \geqslant 1$ имеем

$$
\begin{aligned}
1-a(a-b)(1+a b) & \leqslant 1-(1+a b)=-a b \leqslant 0, \\
1-2 a(a-b)(1+a b) & \leqslant 1-2(1+a b)=-1-2 a b<0 .
\end{aligned}
$$


Значит $k_{2}$ и $k_{1}$ не превосходят нуля и, следовательно, $k_{2} c^{2}+k_{1} c+k_{0}$ убывает по $c$. Поэтому, если при фиксированных $a$ и $b$ уменьшить значение переменной $c, g$ останется положительной. Величину $c$ снизу ограничивают два условия задачи: $a c \geqslant 1$ и $c(b+c) \geqslant 1$. Первое из этих условий избыточно, оно следует из двух других: $c(b+c) \geqslant 1$ и $a(a-b) \geqslant 1$. Действительно, пусть $a c<1$. Тогда

$$
1 \leqslant c(b+c)<\frac{1}{a}\left(b+\frac{1}{a}\right) \Rightarrow a^{2}<a b+1 \Rightarrow a(a-b)<1 .
$$

Значит мы можем уменьшить $c$ до такого значения $c_{*}$, что $c_{*}\left(b+c_{*}\right)=1$. При этом $g$ останется положительной. Но ситуация, когда $c(b+c)=1$ удовлетворяет ограничениям случая $\mathrm{V}$, для которого было доказано, что $g \leqslant 0$. Пришли к противоречию.

Д ок аз а т е л ь с т в о с л е д с т в и я 1. Без ограничения общности считаем $\sigma=1$. Пусть $I$ - случайный индекс, принимающий значения $1, \ldots, n$ с вероятностями $\sigma_{1}^{2}, \ldots, \sigma_{n}^{2}$, не зависящий от $X_{1}, \ldots, X_{n}$. Построим на расширенном вероятностном пространстве

$$
S_{i}^{\prime}:=\sum_{j \neq i} X_{j}+X_{i}^{*}, \quad i=1, \ldots, n,
$$

где $X_{i}^{*}$ имеет распределение $X_{i}$-нулевого смещения и не зависит от $I$, $X_{1}, \ldots, X_{n}, i=1, \ldots, n$. Тогда $S_{n}^{*}=S_{I}^{\prime}$ (см. [19]). Поэтому для произвольной функции $f \in \mathscr{F}_{1}$ имеем

$$
\begin{aligned}
\mathbf{E} f\left(S_{n}^{*}\right) & =\mathbf{E} f\left(S_{I}^{\prime}\right)=\sum_{k=1}^{n} \mathbf{E} f\left(S_{I}^{\prime}\right) \mathbf{1}\{I=k\} \\
& =\sum_{k=1}^{n} \mathbf{E} f\left(S_{k}^{\prime}\right) \mathbf{1}\{I=k\}=\sum_{k=1}^{n} \sigma_{k}^{2} \mathbf{E} f\left(S_{k}^{\prime}\right) .
\end{aligned}
$$

Следовательно,

$$
\begin{aligned}
\left|\mathbf{E} f\left(S_{n}\right)-\mathbf{E} f\left(S_{n}^{*}\right)\right| & =\left|\sum_{k=1}^{n} \sigma_{k}^{2} \mathbf{E} f\left(S_{n}\right)-\sum_{k=1}^{n} \sigma_{k}^{2} \mathbf{E} f\left(S_{k}^{\prime}\right)\right| \\
& \leqslant \sum_{k=1}^{n} \sigma_{k}^{2}\left|\mathbf{E} f\left(S_{n}\right)-\mathbf{E} f\left(S_{k}^{\prime}\right)\right| \\
& \leqslant \sum_{k=1}^{n} \sigma_{k}^{2} \zeta_{1}\left(X_{k}, X_{k}^{*}\right)=\sum_{k=1}^{n} \sigma_{k}^{3} \zeta_{1}\left(\frac{X_{k}}{\sigma_{k}}, \frac{X_{k}^{*}}{\sigma_{k}}\right) \\
& \leqslant \sum_{k=1}^{n} \sigma_{k}^{3} \cdot \frac{1}{2} \mathbf{E}\left|\frac{X_{k}}{\sigma_{k}}\right|^{3}=\frac{1}{2} \sum_{k=1}^{n} \beta_{k}=\frac{1}{2} \varepsilon_{n} .
\end{aligned}
$$

Здесь было использовано утверждение теоремы 3 для с.в. $\sigma_{k}^{-1} X_{k}$, а также свойство однородности метрики $\zeta_{1}$ (т.е. $\zeta_{1}(c X, c Y)=|c| \zeta_{1}(X, Y)$ ) и $(\alpha X)^{*} \stackrel{D}{=} \alpha X^{*}$. 
Доказ а тель с т в о т ео ремы 4. Нетрудно видеть, что для непрерывной $f: \mathbf{R} \rightarrow \mathbf{R}$

$$
h(w):=e^{w^{2} / 2} \int_{-\infty}^{w}(f(w)-\mathbf{E} f(N)) e^{-x^{2} / 2} d x
$$

удовлетворяет уравнению Стейна $h^{\prime}(w)-w h(w)=f(w)-\mathbf{E} f(N)$. Поэтому

$$
\begin{aligned}
\left|\mathbf{E} f\left(S_{n}\right)-\mathbf{E} f(N)\right| & =\left|\mathbf{E} h^{\prime}\left(S_{n}\right)-\mathbf{E} S_{n} h\left(S_{n}\right)\right|=\left|\mathbf{E} h^{\prime}\left(S_{n}\right)-\mathbf{E} h^{\prime}\left(S_{n}^{*}\right)\right| \\
& \leqslant M_{2}(h) \zeta_{1}\left(S_{n}, S_{n}^{*}\right) .
\end{aligned}
$$

Как показано в [20],

$$
M_{2}(h) \leqslant \min \left\{2 M_{1}(f), \frac{\sqrt{2 \pi}}{4} M_{2}(f), \frac{1}{3} M_{3}(f)\right\} .
$$

Принимая во внимание то, что $M_{r}(f) \leqslant 1$ в определении метрики $\zeta_{r}$, имеем

$$
\begin{gathered}
\zeta_{1}\left(S_{n}, N\right) \leqslant 2 \zeta_{1}\left(S_{n}, S_{n}^{*}\right), \quad \zeta_{2}\left(S_{n}, N\right) \leqslant \frac{\sqrt{2 \pi}}{4} \zeta_{1}\left(S_{n}, S_{n}^{*}\right), \\
\zeta_{3}\left(S_{n}, N\right) \leqslant \frac{1}{3} \zeta_{1}\left(S_{n}, S_{n}^{*}\right) .
\end{gathered}
$$

Оценки, содержащие $\varepsilon_{n}$, получаются применением следствия 1 к (28).

Докажем оптимальность (10). Положим $f(x):=x^{3} / 6$. Тогда $M_{3}(f)=1$ и $\mathbf{E} f(N)=0$, поскольку с.в. $N$ симметрична, а функция $f$ нечетна. Пусть $X_{1}, X_{2}, \ldots-$ н.о.р. величины с нулевыми средними и дисперсиями равными 1. Тогда

$$
\mathbf{E} S_{n}^{3}=\frac{\mathbf{E} X_{1}^{3}}{\sqrt{n}}, \quad \varepsilon_{n}=\frac{\mathbf{E}\left|X_{1}\right|^{3}}{\sqrt{n}} .
$$

Следовательно,

$$
\frac{\zeta_{3}\left(S_{n}, N\right)}{\varepsilon_{n}} \geqslant\left|\mathbf{E} f\left(S_{n}\right)-\mathbf{E} f(N)\right|=\frac{1}{6} \frac{\left|\mathbf{E} X_{1}^{3}\right|}{\mathbf{E}\left|X_{1}\right|^{3}} .
$$

Остается доказать, что $\left|\mathbf{E} X_{1}^{3}\right| / \mathbf{E}\left|X_{1}\right|^{3}$ может быть сколь угодно близко к единице. Согласно $(24)$ третий абсолютный момент центрированной с.в. $X_{1}$ с дисперсией 1 , принимающей два значения $x=-\sqrt{q / p}, y=\sqrt{p / q}$ соответственно с вероятностями $p$ и $q$, равен

$$
\mathbf{E}\left|X_{1}\right|^{3}=q \sqrt{\frac{q}{p}}+p \sqrt{\frac{p}{q}} .
$$

нетрудно видеть, что третий момент этой с.в. равен

$$
\mathbf{E} X_{1}^{3}=-q \sqrt{\frac{q}{p}}+p \sqrt{\frac{p}{q}} .
$$

При $p \rightarrow 1$ имеем $\left|\mathbf{E} X_{1}\right| / \mathbf{E}\left|X_{1}\right|^{3} \rightarrow 1$. 
Лемма 1 ([23]). Пусть $W$ - чентрированная с.в. с дисперсией 1 и конечным третьим абсолютным моментом $\beta$. Обозначим $f(t):=$ $\mathbf{E} e^{i t W}, t \in \mathbf{R}$. Тогда при всех $t \in \mathbf{R}$

$$
|f(t)|^{2} \leqslant 1+b(t, \beta+1)
$$

кроме того,

$$
\left|f_{S_{n}}(t)\right|^{2} \leqslant \exp \left(b\left(t, \varepsilon_{n}+\tau_{n}\right)\right) .
$$

Лемма 2. Функиия $b(t, \gamma)$ не убьцвает по $\gamma$ при каждом $t \in \mathbf{R}$.

Д о к а з а т е л с с т в. Проверяется непосредственно вычислением производной.

Следствие 2. Для всех $t \in \mathbf{R} u n \in \mathbf{N}$

$$
\left|f_{S_{n}}(t)\right|^{2} \leqslant \exp \left(b\left(t, 2 \varepsilon_{n}\right)\right)
$$

Д о к а з а т е л ь с т в о. По неравенству Ляпунова имеем $\sigma_{j}^{3} \leqslant \beta_{j}$, $j=1, \ldots, n$. Поэтому $\tau_{n} \leqslant \varepsilon_{n}$. Теперь (31) следует из (30) и леммы 2 .

Лемма 3. При выполнении условий леммы 1

$$
|f(t)-\varphi(t)| \leqslant \varphi(t) \int_{0}^{|t|}\left|f(s)-f^{*}(s)\right| s \exp \left(\frac{s^{2}}{2}\right) d s, \quad t \in \mathbf{R},
$$

где $f^{*}(t)$ - характеристическая функиия с.в. $W^{*}$, имеющей распределение $W$-нулевого смещения.

Д ок аза те ль с т в о. В силу определения распределения $W$ нулевого смещения

$$
\begin{aligned}
f^{\prime}(t) & =i \mathbf{E} W \cos (t W)-\mathbf{E} W \sin (t W) \\
& =-i t \mathbf{E} \sin \left(t W^{*}\right)-t \mathbf{E} \cos \left(t W^{*}\right)=-t \mathbf{E} e^{i t W^{*}} .
\end{aligned}
$$

Рассмотрим функцию $\psi(t):=f(t) / \varphi(t)$. Заметим, что $\psi(0)=1$. $\mathrm{C}$ учетом (33) имеем

$$
\psi^{\prime}(t)=\frac{d}{d t}\left(f(t) e^{\frac{1}{2} t^{2}}\right)=f^{\prime}(t) e^{\frac{1}{2} t^{2}}+t f(t) e^{\frac{1}{2} t^{2}}=\left\{f(t)-f^{*}(t)\right\} t e^{\frac{1}{2} t^{2}} .
$$

Тогда

$$
\left|\frac{f(t)}{\varphi(t)}-1\right|=|\psi(|t|)-\psi(0)| \leqslant \int_{0}^{|t|}\left|\psi^{\prime}(s)\right| d s=\int_{0}^{|t|}\left|f(s)-f^{*}(s)\right| s e^{\frac{1}{2} s^{2}} d s .
$$

Лемма 4. Для любых с.в. $X$ и $Y$ имеем $\left|\mathbf{E} e^{i t X}-\mathbf{E} e^{i t Y}\right| \leqslant|t| \zeta_{1}(X, Y)$. 
Д о к а з а т е л ь с т в о. Хорошо известно, что для любых $t, x, y \in$ $\mathbf{R}$ выполнено неравенство $\left|e^{i t x}-e^{i t y}\right| \leqslant|t| \cdot|x-y|$. Поэтому для любых $\widetilde{X}, \widetilde{Y}$, определенных на одном вероятностном пространстве, таких, что $\operatorname{Law}(\widetilde{X})=\operatorname{Law}(X)$ и $\operatorname{Law}(\tilde{Y})=\operatorname{Law}(Y)$, имеем

$$
\left|\mathbf{E} e^{i t X}-\mathbf{E} e^{i t Y}\right|=\left|\mathbf{E} e^{i t \widetilde{X}}-\mathbf{E} e^{i t \widetilde{Y}}\right| \leqslant \mathbf{E}\left|e^{i t \widetilde{X}}-e^{i t \widetilde{Y}}\right| \leqslant|t| \mathbf{E}|\widetilde{X}-\tilde{Y}| .
$$

Переходя в (34) к нижней грани по всевозможным $\widetilde{X}, \tilde{Y}$, получим

$$
\left|\mathbf{E} e^{i t X}-\mathbf{E} e^{i t Y}\right| \leqslant|t| l_{1}(X, Y)=|t| \zeta_{1}(X, Y) .
$$

Доказ ательст то те о ремы 5. С учетом (31) неравенство (11) тривиально:

$$
\left|f_{S_{n}}(t)-\varphi(t)\right| \leqslant\left|f_{S_{n}}(t)\right|+|\varphi(t)| \leqslant \exp \left(\frac{1}{2} b\left(t, 2 \varepsilon_{n}\right)\right)+\varphi(t) .
$$

Не ограничивая общности, считаем, что $\sigma=1$. Обозначим $f_{j}(t):=$ $\mathbf{E} e^{i t X_{j}}, f_{j}^{*}(t):=\mathbf{E} e^{i t X_{j}^{*}}, j=1, \ldots, n$. Положим в $(32) W:=S_{n}$. Воспользовавшись леммой 4 и следствием 1 , получим

$$
\left|f(s)-f^{*}(s)\right| \leqslant|s| \zeta_{1}\left(S_{n}, S_{n}^{*}\right) \leqslant \frac{\varepsilon_{n}|s|}{2} .
$$

Подставив последнее выражение в (32), приходим к (12).

Согласно (27)

$$
f^{*}(s)=\sum_{m=1}^{n} \sigma_{m}^{2} \mathbf{E} e^{i s S_{m}^{\prime}}=\sum_{m=1}^{n} \sigma_{m}^{2} f_{m}^{*}(s) \prod_{j \neq m} f_{j}(s) .
$$

Следовательно,

$$
\begin{aligned}
\left|f(s)-f^{*}(s)\right| & =\left|\sum_{i=1}^{n} \sigma_{i}^{2} \prod_{j} f_{j}(s)-\sum_{i=1}^{n} \sigma_{i}^{2} f_{i}^{*}(s) \prod_{j \neq i} f_{j}(s)\right| \\
& =\left|\sum_{i=1}^{n} \sigma_{i}^{2}\left\{f_{i}(s)-f_{i}^{*}(s)\right\} \prod_{j \neq i} f_{j}(s)\right|
\end{aligned}
$$

По лемме 4 и теореме 3 имеем

$$
\left|f_{j}(s)-f_{j}^{*}(s)\right| \leqslant|s| \zeta_{1}\left(X_{j}, X_{j}^{*}\right)=|s| \sigma_{j} \zeta_{1}\left(\frac{X_{j}}{\sigma_{j}}, \frac{X_{j}^{*}}{\sigma_{j}}\right) \leqslant \frac{\beta_{j}|s|}{2 \sigma_{j}^{2}} .
$$

Из $(29)$ следует, что $\left|f_{j}(s)\right| \leqslant \exp \left(-\sigma_{j}^{2} s^{2} / 2+2 \beta_{j} a|s|^{3}\right)$ для всех вещественных $s$. Поэтому

$$
\begin{aligned}
\mid f(s) & -f^{*}(s)|\leqslant| \sum_{i=1}^{n} \beta_{i} \frac{s}{2} \prod_{j \neq i} \exp \left(-\frac{\sigma_{j}^{2} s^{2}}{2}+2 \beta_{j} a|s|^{3}\right) \mid \\
& =\left|\sum_{j=1}^{n} \beta_{j} \frac{s}{2} \exp \left(\frac{\sigma_{j}^{2} s^{2}}{2}-2 \beta_{j} a|s|^{3}\right)\right| \exp \left(-\frac{s^{2}}{2}+2 \varepsilon_{n} a|s|^{3}\right) .
\end{aligned}
$$


Поскольку $\sigma_{j}^{3} \leqslant \beta_{j} \leqslant \varepsilon_{n}$ для $j=1, \ldots, n$, имеем для каждого такого $j$ и $t \geqslant 0$

$$
\begin{aligned}
\exp \left(\frac{\sigma_{j}^{2} t^{2}}{2}-2 \beta_{j} a t^{3}\right) & \leqslant \exp \left(\frac{\sigma_{j}^{2} t^{2}}{2}-2 \sigma_{j}^{3} a t^{3}\right)=\left.\exp \left(\frac{s^{2}}{2}-2 a s^{3}\right)\right|_{s=t \sigma_{j}} \\
& \leqslant \sup \left\{\exp \left(\frac{s^{2}}{2}-2 a s^{3}\right): s \in\left[0, t \varepsilon_{n}^{1 / 3}\right]\right\} .
\end{aligned}
$$

Функция $\exp \left(s^{2} / 2-2 a s^{3}\right)$ возрастает на отрезке $[0,1 / 6 a]$, в точке $1 / 6 a-$ глобальный максимум равный $1 / l$. Поэтому

$$
\begin{aligned}
\sup & \left\{\exp \left(\frac{s^{2}}{2}-2 a s^{3}\right): s \in\left[0, t \varepsilon_{n}^{1 / 3}\right]\right\} \\
& = \begin{cases}\exp \left(\frac{t^{2} \varepsilon_{n}^{2 / 3}}{2}-2 a \varepsilon_{n} t^{3}\right), & \text { если } t \varepsilon_{n}^{1 / 3} \leqslant 1 / 6 a ; \\
1 / l, & \text { иначе. }\end{cases}
\end{aligned}
$$

Объединяя (37), (38) и (39), имеем при $|s| \varepsilon_{n}^{1 / 3} \leqslant 1 / 6 a$

$$
\left|f(s)-f^{*}(s)\right| \leqslant \frac{\varepsilon_{n}|s|}{2} \exp \left(\frac{s^{2} \varepsilon_{n}^{2 / 3}}{2}-\frac{s^{2}}{2}\right)
$$

а при $|s| \varepsilon_{n}^{1 / 3}>1 / 6 a$

$$
\left|f(s)-f^{*}(s)\right| \leqslant \frac{\varepsilon_{n}|s|}{2 l} \exp \left(-\frac{s^{2}}{2}+2 a \varepsilon_{n}|s|^{3}\right) .
$$

Подставляя полученные выражения в (32), получим требуемые оценки.

Д о к а з а т ел ь с т в о т е о ре мы 6 . Докажем вначале (14). Пусть $f_{1}(t):=\mathbf{E} e^{i t X_{1}}$. Имеем

$$
\left|f_{S_{n}}(t)-\varphi(t)\right| \leqslant\left|f_{S_{n}}(t)\right|+|\varphi(t)|=\left|f_{1}\left(\frac{t}{\sqrt{n}}\right)\right|^{n}+|\varphi(t)| .
$$

Из леммы 1 следует, что

$$
\begin{aligned}
\left|f_{1}\left(\frac{t}{\sqrt{n}}\right)\right| & \leqslant \sqrt{1+b\left(\frac{t}{\sqrt{n}}, \beta+1\right)}=\sqrt{1+\frac{1}{n} b\left(t, \frac{\beta+1}{\sqrt{n}}\right)} \\
& =\sqrt{1+\frac{1}{n} b\left(t, \varepsilon_{n}+\tau_{n}\right)} .
\end{aligned}
$$

Подставив последнее выражение в (40) получим требуемое утверждение. Чтобы установить (16), заметим, что $1+x \leqslant e^{x}$ при всех вещественных $x$. Применив это неравенство к (14), получим

$$
\left|f_{S_{n}}(t)-\varphi(t)\right| \leqslant \exp \left(\frac{1}{2} b\left(t, \varepsilon_{n}+\tau_{n}\right)\right)+\varphi(t) .
$$


Остается заметить, что последовательность $\left(\tau_{m}\right)_{m \geqslant 1}$ убывает, откуда следует (16).

Положим $W:=S_{n}$ в (32). Воспользовавшись (35) для с.в. $\frac{1}{\sqrt{n}} X_{1}, \ldots, \frac{1}{\sqrt{n}} X_{n}$, получим

$$
\left|f(s)-f^{*}(s)\right|=\left|f_{1}\left(\frac{s}{\sqrt{n}}\right)\right|^{n-1} \cdot\left|f_{1}\left(\frac{s}{\sqrt{n}}\right)-f_{1}^{*}\left(\frac{s}{\sqrt{n}}\right)\right| .
$$

Первый из множителей оцениваем при помощи (41), второй - при помощи (36). Имеем

$$
\left|f(s)-f^{*}(s)\right| \leqslant\left(1+\frac{b\left(s, \varepsilon_{n}+\tau_{n}\right)}{n}\right)^{(n-1) / 2} \frac{\varepsilon_{n}|s|}{2} .
$$

Подставив полученное выражение в (32), получим (15). Для того, чтобы установить (17), применим к первому множителю, стоящему в правой части (42), неравенство $1+x \leqslant e^{x}$ и заметим, что $\left(\tau_{m}\right)_{m \geqslant 1}$ убывает.

Д ок аз а т е ль с т в о т е о р е м ы 7 . Поскольку $\rho\left(S_{n}, N\right) \leqslant 1$, то при $\varepsilon_{n} \geqslant 1 / C$ утверждение теоремы тривиально, поэтому достаточно рассматривать $\varepsilon_{n} \leqslant 1 / 0.6379$ в общем случае, а для н.о.р. величин $\varepsilon_{n} \leqslant$ $1 / 0.5894$. Кроме того, в [24] показано, что при $\varepsilon_{n} \leqslant 1 / 7$

$$
\rho\left(S_{n}, N\right) \leqslant 0.527 \varepsilon_{n} \lambda_{n}^{3 / 2},
$$

а при $\varepsilon_{n} \leqslant 0.1$

$$
\rho\left(S_{n}, N\right) \leqslant 0.5151 \varepsilon_{n} \lambda_{n}^{3 / 2}
$$

где $\lambda_{n}:=\sigma^{2}(n) /\left(\sigma^{2}(n)-\sigma_{m}\right), \sigma_{m}:=\max _{j=1, \ldots, n} \sigma_{j}$. Для н.о.р. слагаемых $\varepsilon_{n}=\beta_{1} /\left(\sigma_{1}^{3} \sqrt{n}\right) \geqslant 1 / \sqrt{n}$. Значит, $n \geqslant\left\lceil 1 / \varepsilon_{n}^{2}\right\rceil$. Поэтому при $\varepsilon_{n} \leqslant 1 / 7$ имеем $\lambda_{n} \leqslant 49 / 48$ и, следовательно,

$$
\rho\left(S_{n}, N\right)<0.5436 \varepsilon_{n} .
$$

В общем случае без ограничения общности считаем $\sigma=1$, тогда $\sigma_{m}^{3} \leqslant$ $\beta_{m} \leqslant \sum_{j=1}^{n} \beta_{j}=\varepsilon_{n}$. Таким образом, при $\varepsilon_{n} \leqslant 0.0484$ выполнено

$$
\begin{aligned}
\rho\left(S_{n}, N\right) & \leqslant 0.5151 \varepsilon_{n}\left(\frac{1}{1-\varepsilon_{n}^{2 / 3}}\right)^{3 / 2} \\
& <0.5151 \varepsilon_{n}\left(\frac{1}{1-0.0484^{2 / 3}}\right)^{3 / 2}<0.6379 \varepsilon_{n} .
\end{aligned}
$$

Следовательно, в общем случае можно ограничиться рассмотрением $\varepsilon_{n}$ из отрезка $I_{1}=[0.0484 ; 1 / 0.6379]$, а в случае н.о.р. величин — из $I_{2}=[1 / 7 ; 1 / 0.5894]$. В основе доказательства теоремы для этих значений $\varepsilon_{n}$ лежит неравенство, полученное В. М. Золотаревым и впоследствии улучшенное ван Биком. 
Пусть $p(u)$ - непрерывная симметричная функция из $L_{1}(\mathbf{R})$, такая, что ее преобразование Фурье $\widehat{p}(t):=\int_{-\infty}^{\infty} e^{i t u} p(u) d u \in L_{1}(\mathbf{R})$,

$$
\begin{gathered}
\|p\|:=\int_{-\infty}^{\infty}|p(u)| d u, \quad v(x):=\int_{0}^{x} \max \{p(u), 0\} d u, \\
q(y):=\frac{1}{\sqrt{2 \pi}} \int_{0}^{\infty}\left|\widehat{p}\left(\frac{t}{y}\right)\right| \frac{\delta_{n}(t)}{t} d t,
\end{gathered}
$$

и существует $\chi_{p}$ - единственный положительный корень уравнения $4 v(x)=\|p\|$. Тогда согласно неравенству ван Бика (см., например, [9])

$$
\frac{\rho\left(S_{n}, N\right)}{\varepsilon_{n}} \leqslant \sqrt{\frac{2}{\pi}} \cdot \frac{v(x) x / y+q(y)}{\varepsilon_{n}(4 v(x)-\|p\|)}, \quad \forall y>0, \quad x>\chi_{p} .
$$

Обозначим

$$
p_{1}(u):=\frac{\sin u}{2 \pi u\left(1-u^{2} / \pi^{2}\right)}, \quad \widehat{p}_{1}(t):=\cos ^{2}\left(\frac{\pi t}{2}\right) \mathbf{1}(|t| \leqslant 1),
$$

где $\mathbf{1}(\cdot)$ - индикаторная функция. Рассмотрим семейство функций

$$
p_{a_{1}, a_{2}, a_{3}}(u):=0.5\left(p_{1}\left(u-a_{1}\right)+p_{1}\left(u+a_{1}\right)+a_{3}\left(p_{1}\left(u-a_{2}\right)+p_{1}\left(u+a_{2}\right)\right)\right),
$$

которым соответствуют преобразования Фурье

$$
\widehat{p}_{a_{1}, a_{2}, a_{3}}(t):=\widehat{p}_{1}(t)\left(\cos \left(a_{1} t\right)+a_{3} \cos \left(a_{2} t\right)\right), \quad a_{1}, a_{2} \in \mathbf{R}, \quad a_{3}>-1 .
$$

Зафиксируем некоторое $\varepsilon>0$. Рассмотрим всевозможные наборы с.в. $X_{1}, \ldots, X_{n}, n \in \mathbf{N}$, такие, что $\varepsilon_{n}=\varepsilon$. Из (43) вытекает, что наименьшая верхняя грань $\rho\left(S_{n}, N\right) / \varepsilon_{n}$ по всем указанным наборам не превосходит при любых значениях параметров $x, y, a_{1}, a_{2}, a_{3}$ величины $D\left(\varepsilon, x, y, a_{1}, a_{2}, a_{3}\right)$, которая получается из правой части $(43)$, если величину $\delta_{n}(t)$, фигурирующую в определении $q(y)$, заменить ее оценкой $\Delta(\varepsilon, t):=\min \left\{\delta^{(1)}(\varepsilon, t), \delta^{(2)}(\varepsilon, t), \delta^{(3)}(\varepsilon, t)\right\}$ и подставить $p:=p_{a_{1}, a_{2}, a_{3}}$ из (44). Для $\varepsilon \in I_{1}$ будем подбирать значения $x, y, a_{1}, a_{2}, a_{3}$ так, чтобы $D\left(\varepsilon, x, y, a_{1}, a_{2}, a_{3}\right)$ оказалась как можно меньше, после чего оценка для $C$ получится взятием верхней грани по всем $\varepsilon \in I_{1}$. Задачу подбора $x$, $y, a_{1}, a_{2}, a_{3}$ можно решать численно при помощи компьютера. В данной работе для этой цели был использован метод сопряженных градиентов, соответствующая процедура взята из библиотеки GNU Scientific Library версии 1.11. Вычисления были проведены для 440 значений $\varepsilon$, для остальных использовано неравенство

$$
D\left(\varepsilon^{(1)}, x, y, a_{1}, a_{2}, a_{3}\right) \leqslant \frac{\varepsilon^{(2)}}{\varepsilon^{(1)}} D\left(\varepsilon^{(2)}, x, y, a_{1}, a_{2}, a_{3}\right), \quad \varepsilon^{(1)}<\varepsilon^{(2)},
$$


которое следует из того, что каждая из функций $\delta^{(1)}(\varepsilon, t), \delta^{(2)}(\varepsilon, t)$, $\delta^{(3)}(\varepsilon, t)$ не убывает по $\varepsilon$. Экстремальное значение $D\left(\varepsilon, x, y, a_{1}, a_{2}, a_{3}\right)=$ 0.637733 возникло при $\varepsilon=0.4767, x=7.5916, y=17.9896, a_{1}=2.2371$, $a_{2}=5.3685, a_{3}=0.5062$.

В случае н.о.р. с.в. использование неравномерных по $n$ оценок позволило добиться лучших результатов. Обозначим $J(\varepsilon, n)$ наименьшую верхнюю грань $\rho\left(S_{n}, N\right) / \varepsilon_{n}$ по всем наборам н.о.p. с.в. $X_{1}, \ldots, X_{n}$ с $\varepsilon_{n}=\varepsilon$. Тогда $C=\sup \{J(\varepsilon, n): \varepsilon>0, n \in \mathbf{N}\}$. Достаточно показать, что $J(\varepsilon, n) \leqslant 0.5894$ при $\varepsilon \in I_{2}, n \geqslant n_{0}(\varepsilon):=\left\lceil 1 / \varepsilon^{2}\right\rceil$. Зафиксируем $\varepsilon$, после чего для нескольких первых значений $n=n_{0}, n_{0}+1$, $\ldots, n_{0}+k$ получим оценку для $J(\varepsilon, n)$ следующим образом. Заменим величину $\delta_{n}(t)$, фигурирующую в определении $q(y)$, оценкой $\Delta_{1}(n, \varepsilon, t):=$ $\min \left\{\delta^{(4)}(n, \varepsilon, t), \delta^{(5)}(n, \varepsilon, t)\right\}$ и возьмем $p=p_{a_{1}, a_{2}, a_{3}}$, после чего в правой части (43) возникнет функция $J_{1}\left(\varepsilon, n, x, y, a_{1}, a_{2}, a_{3}\right)$, при каждом допустимом наборе параметров $x, y, a_{1}, a_{2}, a_{3}$, представляющая собой оценку величины $J(\varepsilon, n)$. Как и ранее, при помощи компьютера подберем $x, y, a_{1}, a_{2}, a_{3}$ так, чтобы $J_{1}\left(\varepsilon, n, x, y, a_{1}, a_{2}, a_{3}\right)$ оказалась как можно меньше.

Для $n>n_{0}+k$ величины $J(\varepsilon, n)$ оценим равномерно. Вместо $\delta_{n}(t)$ подставим $\Delta_{2}(n, \varepsilon, t):=\min \left\{\delta^{(6)}\left(n_{0}+k+1, \varepsilon, t\right), \delta^{(5)}\left(n_{0}+k+1, \varepsilon, t\right)\right\}$. В правой части (43) возникнет функция $J_{2}\left(\varepsilon, x, y, a_{1}, a_{2}, a_{3}\right) \geqslant \sup _{n>n_{0}+k} J(\varepsilon, n)$. Подобрав должным образом $x, y, a_{1}, a_{2}, a_{3}$, найдем искомую оценку. Обозначим $J(\varepsilon)$ максимум из $k+2$ полученных оценок. $J(\varepsilon)$ оценивает сверху величину $\sup _{n} J(\varepsilon, n)$. Поскольку при каждом фиксированном $n$ функции $\delta^{(k)}(n, \varepsilon, t), k=4,5,6,7$, не убывают по $\varepsilon$, то $J(\varepsilon)$ обладает аналогичным (45) свойством, а именно, $J\left(\varepsilon^{(1)}\right) \leqslant\left(\varepsilon^{(2)} / \varepsilon^{(1)}\right) J\left(\varepsilon^{(2)}\right), \varepsilon^{(1)}<\varepsilon^{(2)}$, что позволило проводить вычисления лишь для конечного числа значений $\varepsilon$.

Для случая н.о.р. величин экстремальное значение $J(\varepsilon)=$ $J_{1}\left(\varepsilon, n, x, y, a_{1}, a_{2}, a_{3}\right)=0.589327$ было достигнуто при $\varepsilon=0.14286, n=$ 49 , при этом $x=3.8570, y=28.6444, a_{1}=1.8827, a_{2}=2.8276$, $a_{3}=-0.4959$. Итак, утверждение теоремы справедливо во всех рассмотренных случаях.

Автор считает своим приятным долгом выразить искреннюю признательность профессору А. В. Булинскому за внимание к работе и ценные указания.

\section{СПИСОК ЛИТЕРАТУРЫ}

1. Berry A.C. The accuracy of the Gaussian approximation to the sum of independent variates. - Trans. Amer. Math. Soc., 1941, v. 49, № 1, p. 122-136.

2. Esseen C.-G. On the Liapunoff limit of error in the theory of probability. - Ark. Mat. Astron. Fys., 1942, v. A28, № 9, p. 1-19. 
3. Bergström $H$. On the central limit theorem in the case of not equally distributed random variables. - Skand. Aktuarietidskr., 1949, v. 33, p. 37-62.

4. Takano K. A remark to a result of A. C. Berry. - Res. Mem. Inst. Math., 1951, v. 9, №6, p. 4.08-4.15.

5. Золотарев В. М. О близости распределений двух сумм независимых случайных величин. - Теория вероятн. и ее примен., 1965, т. 10, в. 3, с. 519-526.

6. Золотарев В. М. Абсолютная оценка остаточного члена в центральной предельной теореме. - Теория вероятн. и ее примен., 1966, т. 11, в. 1, с. 108-119.

7. Zolotarev V. M. A sharpening of the inequality of Berry-Esseen. - Z. Wahrscheinlichkeitstheor. verw. Geb., 1967, v. 8, p. 332-342.

8. Золотарев B. M. Некоторые неравенства теории вероятностей и их применение к уточнению теоремы А. М. Ляпунова. - Докл. Акад. наук СССР, 1967, т. 177, B. 3, c. 501-504.

9. van Beek P. An application of Fourier methods to the problem of sharpening the Berry-Esseen inequality. - Z. Wahrsheinlichkeitstheor. verw. Geb., 1972, v. 23, p. $187-196$.

10. Шиганов И. С. Об уточнении верхней константы в остаточном члене центральной предельной теоремы. - Проблемы устойчивости стохастических моделей. Труды ВНИИСИ, 1982, с. 109-115.

11. Шевцова И. Г. Уточнение верхней оценки абсолютной постоянной в неравенстве Берри-Эссеена. - Теория вероятн. и ее примен., 2006, т. 51, в. 3, с. 622-626.

12. Esseen C.-G. A moment inequality with an application to the central limit theorem. Scand. Aktuarietidskr., 1956, v. 39, p. 160-170.

13. Рогозин БА. Одно замечание к работе Эссеена «Моментное неравенство с применением к центральной предельной теореме». - Теория вероятн. и ее примен., 1960 , т. 5 , в. 1 , с. $125-128$.

14. Чистяков Г. П. Новое асимптотическое разложение и асимптотически наилучшие постоянные в теореме Ляпунова. I. - Теория вероятн. и ее примен., 2001, т. 46 , в. 2 , с. $326-344$.

15. Чистяков Г. П. Новое асимптотическое разложение и асимптотически наилучшие постоянные в теореме Ляпунова. II. - Теория вероятн. и ее примен., 2001, т. 46 , в. 3 , с. $573-579$.

16. Чистяков Г. П. Новое асимптотическое разложение и асимптотически наилучшие постоянные в теореме Ляпунова. III. - Теория вероятн. и ее примен., 2002, т. 47, в. 3 , с. $475-497$.

17. Королев В. Ю., Шевцова И. Г. О точности нормальной аппроксимации. I. - Теория вероятн. и ее примен., 2005, т. 50, в. 2, с. 353-366.

18. Королев В.Ю., Шевцова И.Г. О точности нормальной аппроксимации. II. Теория вероятн. и ее примен., 2005, т. 50, в. 3, с. 555-564.

19. Goldstein L., Reinert G. Stein's method and the zero bias transformation with application to simple random sampling. - Ann. Appl. Probab., 1997, v. 7, № 4, p. 935-952.

20. Raic M. Normal approximation by Stein's method. Proceedings of the Seventh Young Statisticians Meeting. - Metodoloski zvezki, 2003, v. 21, p. 71-97.

21. Hoeffding $H$. The extrema of the expected value of a function of independent random variables. - Ann. Math. Statist., 1955, v. 26, № 2, p. 268-275.

22. Золотарев B. M. Современная теория суммирования независимых случайных величин. М.: Наука, 1986.

23. Prawitz $H$. Weitere Ungleichungen fur den absoluten Betrag einer charakteristischen Function. - Scand. Actuarial J., 1974, p. 21-28.

24. Prawitz H. On the remainder in the central limit theorem. - Scand. Actuarial J., 1975 , p. $145-156$. 